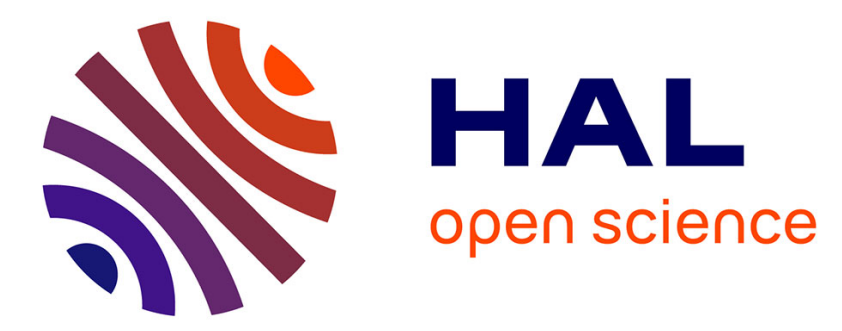

\title{
Mobility of domain wall motion in the permalloy layer of a spin-valve-like $\mathrm{Fe} 20 \mathrm{Ni} 80 / \mathrm{Cu} / \mathrm{Co}$ trilayer
}

Keiki Fukumoto, Wolfgang Kuch, Jan Vogel, Julio Camarero, Stefania Pizzini, Francesco Offi, Yan Pennec, Marlio Bonfim, Alain Fontaine, Jürgen Kirschner

\section{To cite this version:}

Keiki Fukumoto, Wolfgang Kuch, Jan Vogel, Julio Camarero, Stefania Pizzini, et al.. Mobility of domain wall motion in the permalloy layer of a spin-valve-like $\mathrm{Fe} 20 \mathrm{Ni} 80 / \mathrm{Cu} / \mathrm{Co}$ trilayer. Journal of Magnetism and Magnetic Materials, 2005, 293, pp.863. 10.1016/j.jmmm.2004.12.009 . hal-00004890

\section{HAL Id: hal-00004890 \\ https://hal.science/hal-00004890}

Submitted on 10 May 2005

HAL is a multi-disciplinary open access archive for the deposit and dissemination of scientific research documents, whether they are published or not. The documents may come from teaching and research institutions in France or abroad, or from public or private research centers.
L'archive ouverte pluridisciplinaire HAL, est destinée au dépôt et à la diffusion de documents scientifiques de niveau recherche, publiés ou non, émanant des établissements d'enseignement et de recherche français ou étrangers, des laboratoires publics ou privés. 


\title{
Mobility of domain wall motion in the permalloy layer of a spin-valve-like $\mathrm{Fe}_{20} \mathrm{Ni}_{80} / \mathrm{Cu} / \mathrm{Co}$ trilayer
}

\author{
K. Fukumoto ${ }^{1 *}$, W. Kuch ${ }^{1 *}$, J. Vogel ${ }^{2}$, J. Camarero ${ }^{3}$, S. Pizzini ${ }^{2}$, F. Offi ${ }^{1 \dagger}$, Y. Pennec ${ }^{2}$, \\ M. Bonfim ${ }^{4}$, A. Fontaine ${ }^{2}$, and J. Kirschner ${ }^{1}$ \\ ${ }^{1}$ Max-Planck-Institut für Mikrostrukturphysik, Weinberg 2, D-06120 Halle, Germany \\ ${ }^{2}$ Laboratoire Louis Néel, CNRS, 25 avenue des Martyrs, B. P. 166, F-38042 Grenoble Cedex 9, France \\ ${ }^{3}$ Departamento de Física de la Materia Condensada, Universidad Autónoma de Madrid, E-28049 Madrid, \\ Spain \\ ${ }^{4}$ Departamento de Engenharia Elétrica, Universidade do Paraná, CEP 81531-990, Curitiba, Brazil
}

\begin{abstract}
The magnetization reversal of the magnetically soft permalloy $\left(\mathrm{Fe}_{20} \mathrm{Ni}_{80}\right)$ layer in a spin-valve like $\mathrm{FeNi} / \mathrm{Cu} / \mathrm{Co}$ trilayer was studied using photoelectron emission microscopy combined with $\mathrm{x}$-ray magnetic circular dichroism, which allows to image the magnetic domain structure in an element-selective way. Nanosecond-short magnetic field pulses with amplitudes between 3.3 and $16.3 \mathrm{mT}$ were applied one by one to reverse the magnetization of the FeNi layer. Images of the magnetic domain structure were taken after application of each pulse, and the mobility of magnetic domain wall motion in the FeNi layer was deduced. The reversal mechanism of the FeNi layer was found to depend on the applied pulse length, amplitude, and on the energy and direction of the coupling between the two ferromagnetic layers.
\end{abstract}




\section{Introduction}

Nowadays, magnetic recording devices contain magnetic trilayered systems, so called spin valves (SV) or magnetic tunnel junctions (MTJ), in which two ferromagnetic (FM) layers are separated by a non-magnetic spacer layer. These devices are operating in the $\mathrm{GHz}$ range. Their magnetization reversal processes, however, are not well known because of the complexity of the properties of magnetic multilayers, like the dependence of magnetic coupling on interlayer thickness, surface/interface roughness, and amplitude and sweep rate of the external field. For a detailed understanding of the dynamic magnetic switching of this kind of trilayers, it is important to investigate the switching behavior of the two FM layers separately. In this paper we study the magnetization reversal of an $\mathrm{FeNi} / \mathrm{Cu} / \mathrm{Co}$ SV using element-selective photoelectron emission microscopy (PEEM) measurements.

The magnetization reversal of single FM layers has been widely investigated by time-resolved Kerr microscopy [1-3] and scanning electron microscopy with polarization analysis [4], in a temporal range from several seconds down to the picosecond [5, 6]. Dynamic coercivity measurements have also been carried out to obtain information on the mechanism of magnetization reversal [7].

In films in which domain wall motion dominates the reversal in the quasi-static regime, it was shown that at low sweep rate of the external field, $\mathrm{H}_{E x t}$, the coercive field increases linearly with the logarithm of $\mathrm{dH}_{E x t} / \mathrm{dt}$ [7-9]. The speed of magnetization reversal increases exponentially with $\mathrm{H}_{E x t}$ below a certain value of the critical field, $\mathrm{H}_{C r i t}$, which is related to the height of the energy barrier that pins domain wall motion. In this regime, the domain wall propagates step-by-step. It is blocked at an energy barrier, caused for example by surface roughness, until it acquires enough energy (by thermal activation or increasing $\mathrm{H}_{E x t}$ ) to overcome the barrier and progress to the next one. If $\mathrm{H}_{E x t}$ is well above $\mathrm{H}_{C r i t}$, the domain wall speed increases linearly with $\mathrm{H}_{E x t}$. The exponential and linear regimes correspond to thermally activated and viscous domain wall motion, respectively [10]. Estimated speeds of wall motion were around a few hundred $\mathrm{m} / \mathrm{s}$ or slower in the former regime for samples with perpendicular magnetic anisotropy [11, 12]. The speed of wall motion in the viscous regime was studied for FeNi layers with inplane anisotropy [13-15]. In general, for high values of the magnetic field sweep rate (above about $100 \mathrm{~T} / \mathrm{s}$ in the SV sample studied here), magnetic domain nucleation starts to dominate the reversal, and the coercive field increases exponentially as a function of $\mathrm{dH}_{E x t} / \mathrm{dt}$ [7-9].

The element selective magnetization dynamics of magnetic multi-layered sys- 
tems (SV and MTJ) has already been studied in the ns range using time-resolved x-ray magnetic circular dichroism measurements [16]. With these measurements, the global magnetization reversal of each of the layers after application of ns long pulses could be obtained. In this contribution, the magnetization reversal of the magnetically soft permalloy $\left(\mathrm{Fe}_{20} \mathrm{Ni}_{80}, \mathrm{FeNi}\right.$ in the following) layer in a SV system was studied using PEEM with $\mathrm{x}$-ray magnetic circular dichroism (XMCD-PEEM), which allows element selective magnetic domain imaging with sub-micron resolution. A dependence of the magnetization reversal process of the FeNi layer on the magnetization direction of the hard Co layer is observed. 


\section{Experiment}

The SV sample, $5 \mathrm{~nm} \mathrm{Fe}{ }_{20} \mathrm{Ni}_{80} / 10 \mathrm{~nm} \mathrm{Cu} / 5 \mathrm{~nm}$ Co capped with $1.5 \mathrm{~nm}$ of $\mathrm{Au}$, was grown by molecular beam epitaxy on a step-bunched $\mathrm{Si}(111)$ surface. The $\mathrm{Si}$ surface was protected by capping with a $0.3 \mathrm{~nm} \mathrm{Cu}$ layer. The miscut of the surface was $4^{\circ}$ along the $[11 \overline{2}]$ crystal axis. The substrate surface presents terraces (approximate dimensions $1 \mu \mathrm{m} \times 60 \mathrm{~nm}$ ) that are transferred to the magnetic films. The two FM layers exhibit in-plane uniaxial anisotropy with the easy axes along the step bunches. Magnetostatic coupling is concentrated at the step-bunches. More details on the preparation and magnetic properties of this kind of samples can be found in Refs. 7 and 17.

The magnetic domain structure in the FeNi layer was measured using XMCDPEEM with the $\mathrm{x}$-ray photon energy tuned to the $\mathrm{Fe}-\mathrm{L}_{3}$ absorption edge. Magnetic domain structure images were created taking the difference divided by the sum of two images that were taken with positive and negative helicity of circularly polarized x-rays. The lateral resolution was set to $1 \mu \mathrm{m}$ in this experiment. The grey scale contrast of the PEEM images then depends on the angle between the direction of the incoming circularly polarized x-rays and the local magnetization direction in the film. The PEEM and the measuring geometry used in this study is identical to the one described elsewhere [18]. The measurements were performed on beamline UE56-2/PGM2 of the synchrotron BESSY in Berlin.

To study the magnetization reversal properties of the FeNi layer magnetically coupled to the Co layer, first both ferromagnetic layers were saturated either in the same or in opposite directions. $1 \mathrm{~ms}-$ long, $30 \mathrm{mT}$ magnetic pulses applied along the easy axis were strong enough to saturate both films, and a subsequent $4.5 \mathrm{mT}$ field reversed the magnetization of only the FeNi layer. $30 \mathrm{~ns}$ and 120 ns-short field pulses with various amplitudes were then applied to reverse the magnetization of the FeNi layer. The shape of these pulses is shown in Fig. 1. They were produced by a handmade micro-coil directly mounted on the sample. The field amplitude was calibrated by Faraday effect measurements with a paramagnetic sample, and the error was estimated to be $\pm 10 \%$, as described in Ref. 19. Images of the static magnetic domain structure were taken after the application of each magnetic pulse, so called single-pulse measurements. Stroboscopic images of the dynamics of the magnetization reversal during the pulses are presented in other papers [19-21]. 
Results

In the top graph of Fig. 2, the magnetization curve of the SV system obtained by quasi-static Kerr effect measurements is shown by the grey solid line. A double step reversal being due to separate switching of the FeNi layer (lower coercivity) and the Co layer (higher coercivity) is seen. One of the two minor loops of the FeNi layer is also shown by the grey dashed curve, where the interlayer coupling is evidenced by the horizontal shift of the minor loop. The coupling, $\mathrm{H}_{\text {coupl }}$, of about $2 \mathrm{mT}$ is caused by correlated roughness at the two $\mathrm{FM} / \mathrm{Cu}$ interfaces leading to a parallel coupling between two FM layers [22]. A localization of the coupling at the steps was found in the same type of sample [23]. The images in Fig. 2 (a) to (f) show the magnetic domain structure of the FeNi layer induced by 30 ns-short pulses (dashed curve in Fig. 1). The scale of the images is indicated on (d).

First, both FM layers were saturated in parallel along the easy axis of magnetization, parallel to the step-bunches, by applying $30 \mathrm{mT}$ field pulses of $1 \mathrm{~ms}$ with an external coil (configuration A). Saturation was checked by XMCD-PEEM domain images. Magnetic pulses were then applied by the micro coil in the direction opposite to the magnetization directions of the FeNi and Co layers.

PEEM image (a) was taken after application of 31 pulses of $4.8 \mathrm{mT}$. By applying these pulses, a white domain appeared from the left side of the image and propagated to the right. The direction of the field pulses and the magnetization directions of white and black regions are indicated by arrows. In general, domain wall motion as observed here is the predominant mechanism if the amplitude of the applied field is slightly higher than the coercive field, and also the zig-zag shaped domain wall is typical for head-on walls in films with uniaxial in-plane anisotropy [24]. Applying one $9.0 \mathrm{mT}$ pulse after $r$-saturation of the films, also magnetic domain nucleation was observed and $55 \%$ of the image turned to white, as shown in Fig. 2(b). In Fig. 2(c), an image is shown taken after application of one $16.3 \mathrm{mT}$ pulse, leading to the nucleation of many small domains. Some of these domains were smaller than the lateral resolution, leading to an intermediate grey contrast. In summary, by increasing the pulse amplitude, a gradual transition of the reversal process from domain wall propagation to domain nucleation is observed. This leads to an increase of the number of domains, and a decrease of their average size with pulse amplitude.

In a second experiment, the two FM layers were initially saturated in opposite directions (configuration B), and field pulses were then applied in the same direction as the Co layer magnetization. For single pulses, the magnetic fields needed to reverse the FeNi magnetization in configuration B are much smaller than for 
configuration A. In configuration B, after one field pulse, domains were observed in a narrow range of fields, between 4.0 and $5.0 \mathrm{mT}$. Totally black (white) images were observed below $4.0 \mathrm{mT}$ (above $5.0 \mathrm{mT}$ ) (images are not shown in Fig. 2). Domain wall propagation dominates the reversal. The percentage of the FeNi magnetization reversed in the PEEM images for different applied pulse fields is also plotted in the top graph in Fig. 2, using the right axis. The dashed black lines are guides to the eye through the experimental points, indicating the FeNi hysteresis curve obtained for 30 ns-short field pulses for the two directions of the Co magnetization. The experimental points (a)-(f) are indicating the field amplitude of the pulses after which the corresponding images have been taken in remanence and the corresponding permalloy magnetization. Static Kerr effect measurements performed on a different piece of the same sample show that the magnetization does not change when the field is decreased to zero on different points during the switching of the FeNi layer, i.e. that the switching process is irreversible. This indicates that, at least under quasi-static conditions, after switching off the external magnetic field there is no domain wall motion induced by the coupling to the Co layer. From Fig. 2 it is obvious that the FeNi reversal for switching parallel to the magnetization direction of the Co layer and antiparallel to it is quite different. This different behavior will be discussed below.

Single-pulse measurements were also performed using $120 \mathrm{~ns}$-short pulses with $10 \mathrm{~ns}$ rise- and drop-time (solid curve in Fig. 1). Panels (a) to (g) in Fig. 3 show the magnetic domain structure of the FeNi layer after successive application of 120 ns field pulses of identical amplitude. The field of view is $120 \mu \mathrm{m}$. Starting from configuration A, pulses with amplitudes of $5.0 \mathrm{mT}$ (i) and $6.1 \mathrm{mT}$ (ii), were applied to reverse the magnetization of the FeNi layer. One $5.0 \mathrm{mT}$ pulse created a small white domain at the middle of the image (a) which was probably nucleated at a surface defect. Then a second pulse was applied without re-saturation of the film, and a white zig-zag shaped domain appeared on the left side (b). The third pulse made the domain wall propagate to the right (c). Domain wall propagation was also observed when applying pulses of $6.1 \mathrm{mT}$ (ii) and $3.3 \mathrm{mT}$ (iii). In (iii), the direction of the external field was parallel to the Co magnetization (configuration B). The magnetization of the Co layer was not influenced by the pulses with the amplitudes used here.

The speed of domain wall motion of the FeNi layer was estimated from these images in the following way. White circles and crosses were put onto the tips of white zig-zag domains in (b), (d) and (f) and (c), (e) and (g), respectively. The circles were superimposed from (b), (d) and (f) to (c), (e) and (g), respectively. 
By taking the average displacement of the tips, the estimated speed of wall motion was $270 \mathrm{~m} / \mathrm{s}, 410 \mathrm{~m} / \mathrm{s}$ and $490 \mathrm{~m} / \mathrm{s}$ for the three field pulses. In (ii) from (d) to (e), the shape of the domains was more or less preserved, but not in (i) and (iii). This leads to different errors in the domain wall velocity of $\pm 20 \mathrm{~m} / \mathrm{s}$ for (ii) and (iii), and $\pm 80 \mathrm{~m} / \mathrm{s}$ for (i). However, we can not exclude that some new domains nucleated between (d) and (e) since some small black domains are visible in the white region. In configuration $\mathrm{B}$ where the magnetization of FeNi reverses into the direction of the Co layer, faster wall propagation as compared with configuration A was observed with a smaller field pulse amplitude. 


\section{Discussion}

In Fig. 2, it was shown that the magnetization reversal of the FeNi layer for switching into directions anti-parallel (configuration A) or parallel (configuration B) to the Co magnetization direction is quite different. For configuration A, a transition in magnetization reversal mechanism was observed when $\mathrm{H}_{E x t}$ was increased from 4.8 to $16.3 \mathrm{mT}$. For the higher amplitude of pulses the density of nucleation centers increases and the domain size decreases. For configuration $\mathrm{B}$, observations could be made only over a much narrower field range, and no significant difference in the density of nucleation centers could be deduced from the images

The magnetic coupling is shown to play an important role in these measurements. The same effective field pulses $\left(\mathrm{H}_{e f f}=\mathrm{H}_{E x t} \pm \mathrm{H}_{\text {coupl }}\right)$ applied to the sample initially in configurations $\mathrm{B}$ or A give rise to different magnetization reversal processes. In figure 2 (b) and (f) the same $\mathrm{H}_{e f f}$ (around $7.0 \mathrm{mT}$ ) is applied, but the domain configuration after application of one pulse is very different. While it is difficult to conclude about differences in nucleation densities in the two cases, it is clear that in configuration $\mathrm{B}$, where the FeNi layer is switched towards the Co magnetization direction, domain wall propagation is easier than in configuration A. When the magnetization of the FeNi layer is switched against the Co magnetization direction (configuration A), the coupling, concentrated at the steps between terraces, causes them to act as blocking centers for domain wall motion [7].

To estimate the domain wall velocity, v, as a function of field using Fig. 3, $\mathrm{H}_{\text {coupl }}$ has to be taken into account. The speed of domain wall motion as a function of $\mathrm{H}_{e f f}$ is shown in Fig. 4. In cases (i) and (ii), $\mathrm{H}_{\text {coupl }}(=2.0 \mathrm{mT})$ is against the pulse field, therefore $\mathrm{H}_{e f f}=\mathrm{H}_{E x t}-\mathrm{H}_{\text {coupl }}$. This results in effective fields $\mathrm{H}_{\text {eff }}=$ $3.0 \mathrm{mT}$ and $4.1 \mathrm{mT}$ for (i) and (ii), respectively. In (iii), $\mathrm{H}_{\text {eff }}=\mathrm{H}_{E x t}+\mathrm{H}_{\text {coupl }}$ was $5.3 \mathrm{mT}$, with $\mathrm{H}_{E x t}=3.3 \mathrm{mT}$. In this experiment, domain wall motion is expected to be in the viscous regime [10], since $\mathrm{H}_{e f f}$ is well above $\mathrm{H}_{\text {Crit }}$ for all three cases. The value of $\mathrm{H}_{\text {Crit }}$ is estimated to be about $2 \mathrm{mT}$. This value was obtained using equation (3) of Ref. 25 which relates $\mathrm{E}_{A}$, the activation energy, to $\mathrm{H}_{C r i t}$. $\mathrm{E}_{A}$ should be equal to $\mathrm{V}_{B} \mathrm{M}_{S} \mathrm{H}_{C r i t}$ without external field, where $\mathrm{V}_{B}$ and $\mathrm{M}_{S}$ are the Barkhausen volume and the saturation magnetization of the FeNi layer, respectively. $\mathrm{E}_{A}$ and $\mathrm{V}_{B}$ were obtained from $\mathrm{H}$ vs.dH/dt measurements [26], giving $2.98 \times 10^{-19} \mathrm{~J}(1.86$ $\mathrm{eV})$ and $1.88 \times 10^{-22} \mathrm{~m}^{3}$, respectively. $\mathrm{M}_{S}$ was taken as $1 \mathrm{~T}\left(800 \mathrm{emu} / \mathrm{cm}^{3}\right)$.

In the viscous regime, the speed of domain wall motion increases linearly with $\mathrm{H}_{e f f}$. Our data are well fitted using $\mathrm{v}=\mu \mathrm{H}_{e f f}$, where $\mu$ is the domain wall mobility. The obtained value of $\mu$ is $100 \pm 10 \mathrm{~m} \mathrm{~s}^{-1}(\mathrm{mT})^{-1}$. For 30 and $10 \mathrm{~nm}$-thick FeNi 
layers, values of $380 \mathrm{~m} \mathrm{~s}^{-1}(\mathrm{mT})^{-1}$ and $300 \mathrm{~m} \mathrm{~s}^{-1}(\mathrm{mT})^{-1}$ have been reported, respectively $[14,15]$. It is supposed that by decreasing the film thickness the mobility decreases. Another possible explanation is the large roughness of our sample due to the steps with a height of about $6 \mathrm{~nm}$. This roughness may lead to a domain wall mobility that is lower than in flat films.

The domain wall mobility depends on the damping parameter $\alpha$ and the domain wall width of the permalloy layer, since $\mu=\gamma \Delta / \alpha$ [10], where $\gamma$ is the gyromagnetic ratio $\left(=1.79 \times 10^{11} \mathrm{~T}^{-1} \mathrm{~s}^{-1}\right)$ and $\Delta$ is the domain wall width parameter. In the case of a Bloch wall, $\Delta$ equals the wall width [27]. For a layer thickness of $5 \mathrm{~nm}$, the domain walls in the FeNi layer are supposed to be the Néel type [14, 15, 28]. In the case of a Néel wall the relation between the wall width and the domain wall width parameter $\Delta$ is not known, and an evaluation of the damping parameter $\alpha$ from the present measurements is therefore limited to a rough order-of-magnitude estimate. Setting the wall width parameter $\Delta$ equal to the exchange length of FeNi (about $6 \mathrm{~nm}$ ), a value of 0.01 for $\alpha$ results. This is reasonable considering other experimental values, which range from 0.008 to 0.013 for FeNi film thicknesses between 10 and $50 \mathrm{~nm}$ [29-33]. 


\section{Conclusion}

The magnetization reversal behavior of the soft FeNi layer, magnetically coupled to a Co layer through a $\mathrm{Cu}$ spacer layer in a spin-valve-like trilayer, was studied using XMCD-PEEM in the ns range.

A difference was observed in the reversal mechanism of the FeNi layer when its magnetization direction was switched into the direction parallel or anti-parallel to the Co magnetization direction. In the former case, reversal by domain wall propagation was dominant, while in the latter case domain nucleation played a larger role for higher field amplitudes.

The magnetic domain wall motion in the FeNi layer was studied in the viscous regime, in which the domain wall velocity increases linearly as a function of the effective field. The slope of this function gives the mobility of wall motion, which was $100 \mathrm{~m} \mathrm{~s}^{-1}(\mathrm{mT})^{-1}$ in this study.

\section{Acknowledgment}

We thank F. Helbig, B. Zada, and W. Mahler for technical support. We also thank F. Petroff and A. Vaurès for sample preparation. Financial support by BMBF (no. 05KS1EFA6) and EU (BESSY-EC-HPRI Contract No. HPRI-1999-CT-00028) is gratefully acknowledged. 


\section{References}

* Present address: Freie Universität Berlin Institut für Experimentalphysik, Arnimallee 14, D-14195 Berlin, Germany.

$\dagger$ Present address: INFM and Dipartimento di Fisica, Universita' Roma Tre, Via della Vasca Navale 84, I-00146 Roma, Italy.

1. M. Labrune, S. Andrieu, F. Rio and P. Bernstein, J. Magn. Magn. Mater., 80 (1989) 211.

2. A. Kirilyuk, J. Giergiel, J. Shen and J. Kirschner, J. Magn. Magn. Mater., 159 (1996) L27.

3. W. Y. Lee, B.-Ch. Choi, Y. B. Xu and J. A. C. Bland, Phys. Rev. B, 60 (1999) 10216.

4. E. Mivehchi, D. H. Horrocks, H. Iranmanesh and P. Beckley, J. Magn. Magn. Mater., 133 (1994) 405.

5. M. R. Freeman and J. F. Smyth, J. Appl. Phys., 79 (1996) 5898.

6. R. H. Koch, J. G. Deak, D. W. Abraham, P. L. Trouilloud, R. A. Altman, Yu Lu, W. J. Gallagher, R. E. Scheuerlein, K. P. Roche and S. S. P. Parkin, Phys. Rev. Lett., 81 (1998) 4512.

7. Y. Pennec, J. Camarero, J. C. Toussaint, S. Pizzini, M. Bonfim, F. Petroff, W. Kuch, F. Offi, K. Fukumoto, F. Nguyen Van Dau and J. Vogel, Phys. Rev. B, 69 (2004) 180402(R).

8. T. A. Moore, J. Rothman, Y. B. Xu and J. A. C. Bland, J. Appl. Phys., 89 (2001) 7018.

9. I. Ruiz-Feal, T. A. Moore, L. Lopez-Diaz and J. A. C. Bland, Phys. Rev. B, 65 (2002) 54409.

10. J. Ferré in Spin Dynamics in Confined Magnetic Structure I, eds. B. Hillebrands and K. Ounadjela, Topics Appl. Phys. 83 (Springer, Berlin, Heidelberg 2002), P127.

11. S. Lemerle, J. Ferré, C. Chappert, V. Mathet, T. Giamarch and P. Le Doussal, Phys. Rev. Lett., 80 (1998) 849.

12. A. Kirilyuk, J. Ferré, V. Grolier, J. P. Jamet and D. Renard, J. Magn. Magn. Mater., 171 (1997) 45. 
13. S. W. Yuan and H. Neal Bertram, Phys. Rev. B, 44 (1991) 12395.

14. S. Konishi, S. Yamada and T. Kosuda, IEEE Trans. Magn., 7 (1971) 722.

15. M. Redjdal, J. Giusti, M. F. Ruane and F. B. Humphrey, J. Appl. Phys., 91 (2002) 7547.

16. M. Bonfim, G. Ghiringhelli, F. Montaigne, S. Pizzini, N. B. Brookes, F. Petroff, J. Vogel, J. Camarero and A. Fontaine, Phys. Rev. Lett., 86 (2001) 3646.

17. A. Encinas, F. Nguyen Van Dau, M. Sussiau, A. Schuhl and P. Galtier, Appl. Phys. Lett., 71 (1997) 3299.

18. K. Fukumoto, H. Daimon, L. I. Chelaru, F. Offi, W. Kuch and J. Kirschner, Surf. Sci., 514 (2002) 151.

19. J. Vogel, W. Kuch, J. Camarero, K. Fukumoto, Y. Pennec, M. Bonfim, S. Pizzini, F. Petroff, A. Fontaine and J. Kirschner, J. Appl. Phys., 95 (2004) 6533.

20. J. Vogel, W. Kuch, M. Bonfim, J. Camarero, Y. Pennec, F. Offi, K. Fukumoto, J. Kirschner, A. Fontaine and S. Pizzini, Appl. Phys. Lett., 82 (2003) 2299.

21. W. Kuch, J. Vogel, J. Camarero, K. Fukumoto, Y. Pennec, S. Pizzini, M. Bonfim and J. Kirschner, Appl. Phys. Lett., 85 (2004) 440.

22. L. Néel, C.R. Acad. Sci. Paris 255 (1962) 1676.

23. Y. Pennec, Ph. D. thesis, Université Joseph Fourier, Grenoble, France, 2003

24. A. Hubert and R. Schäfer, Magnetic Domains, (Springer, 1998).

25. P. Bruno, G. Bayreuther, P. Beauvillain, C. Chappert, G. Lugert, D. Renard, J. P. Renard and J. Seiden, J. Appl. Phys., 68 (1990) 5759.

26. J. Camarero, Y. Pennec, J. Vogel, M. Bonfim, S. Pizzini, M. Cartier, F. Ernult, F. Fetter and B. Dieny, Phys. Rev. B, 64 (2001) 172402.

27. J. S. Dillon, Jr., Magnetism, Vol. 3, Ch. 9, Academic Press, 1963

28. T. Trunk, M. Redjdal, A. Kákay, M. F. Ruane and F. B. Humphrey, J. Appl. Phys., 89 (2001) 7606.

29. W. K. Hiebert, A. Stankiewicz and M. R. Freeman, Phys. Rev. Lett., 79 (1997) 1134. 
30. G. M. Sandler, H. N. Bertram, T. J. Silva and T. M. Crawford, J. Appl. Phys., 85 (1999) 5080.

31. Th. Gerrits, J. Hohlfeld, O. Gielkens, K. J. Veenstra, K. Bal, Th. Rasing and H. A. M. van den Berg, J. Appl. Phys., 89 (2001) 7648.

32. J. P. Nibarger, R. Lopusnik, Z. Celinski and T. J. Silva, Appl. Phys. Lett., 83 (2003) 93.

33. S. Ingvarsson, L. Ritchie, X. Y. Liu, Gang Xiao, J. C. Slonczewski, P. L. Trouilloud and R. H. Koch, Phys. Rev. B, 66 (2002) 214416. 


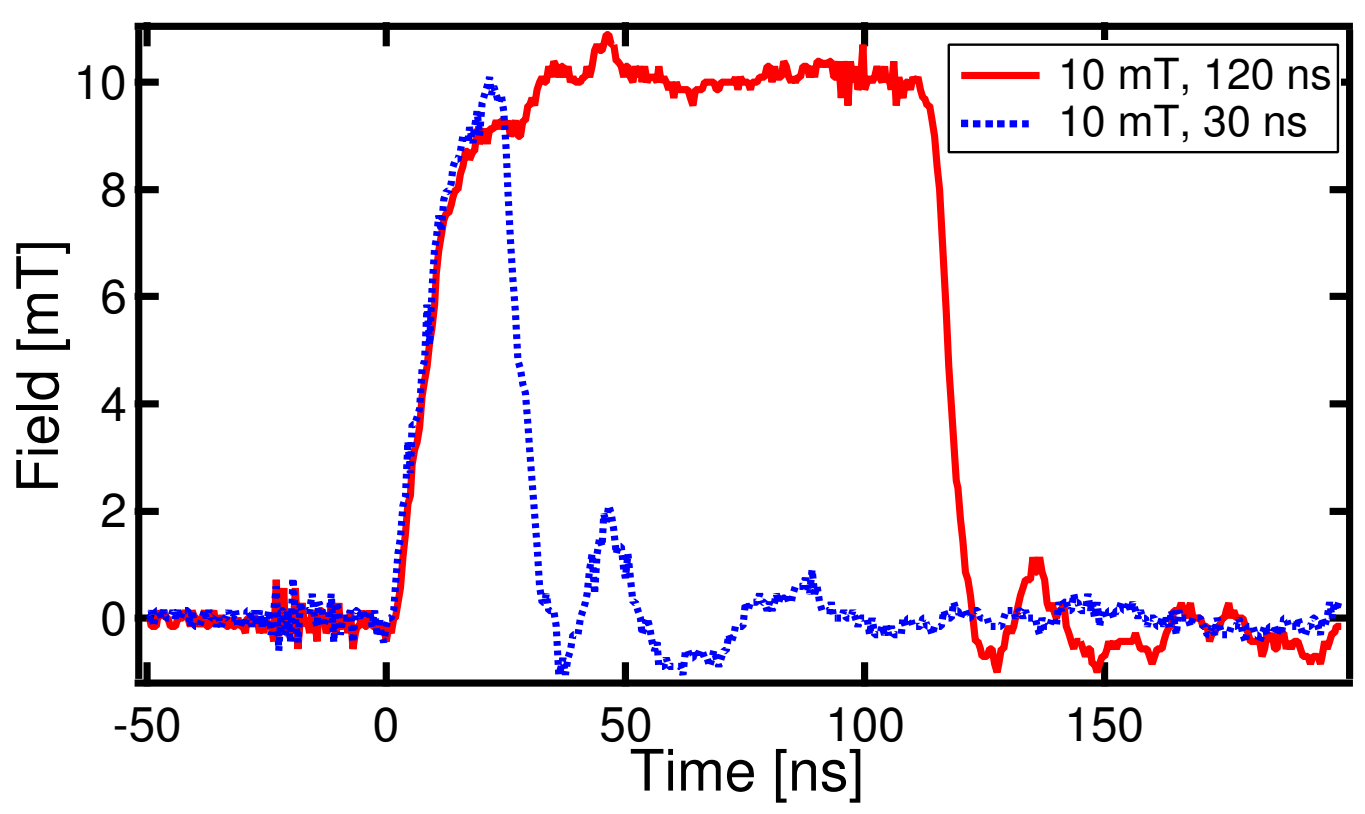

Figure 1: Shape of the magnetic field pulses. Solid and dotted curves correspond to $120 \mathrm{~ns}$ and 30 ns pulses, respectively. The height of both pulses shown here is $10 \mathrm{mT}$. 

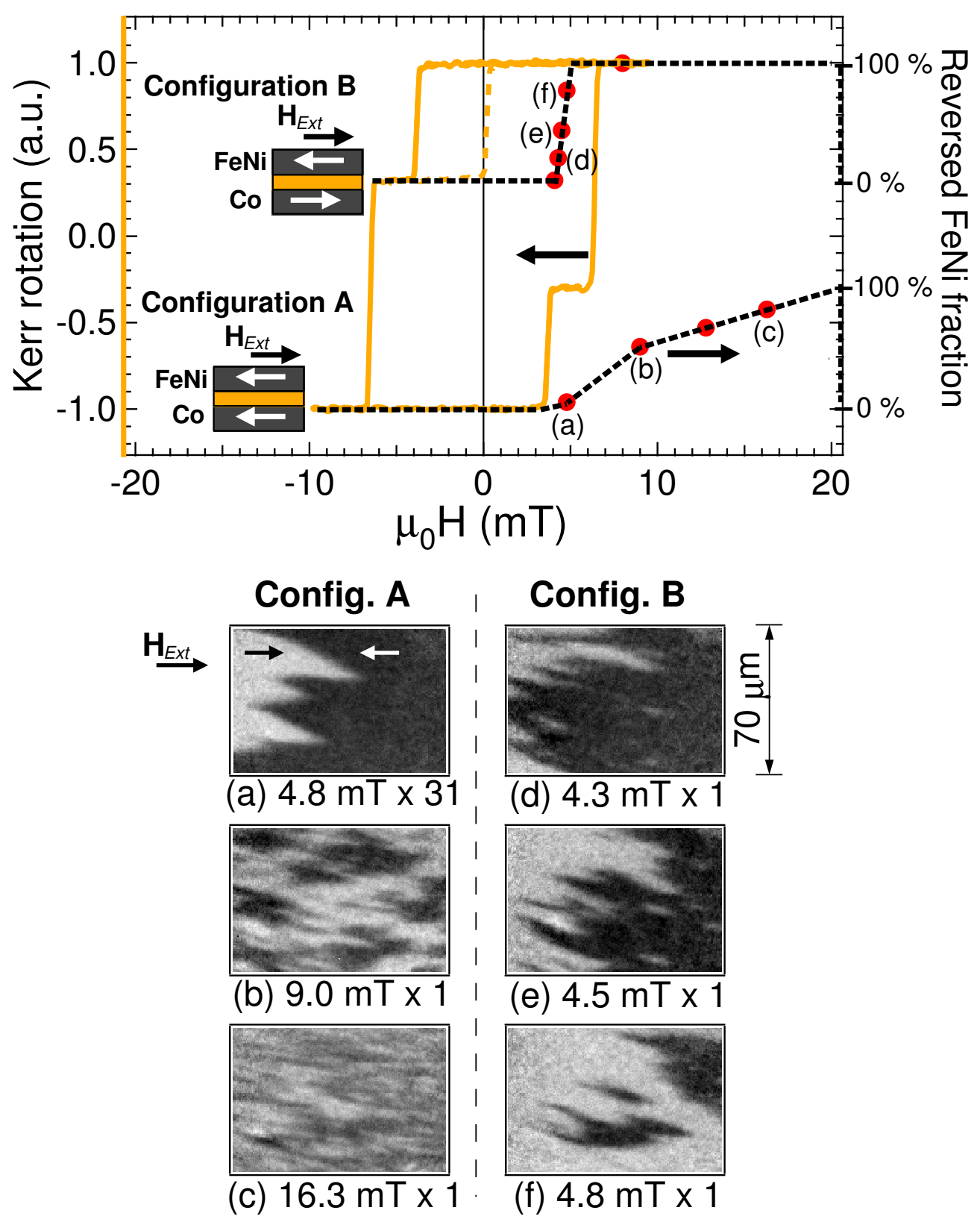

Figure 2: Hysteresis loop obtained by longitudinal Kerr effect measurements of the $\mathrm{FeNi} / \mathrm{Cu} / \mathrm{Co}$ trilayer (grey solid curve) in quasi-static conditions. One of two minor loops of the FeNi soft layer is also shown (grey dotted curve). The normalized Kerr rotation is labeled on the left axis. Black dotted curves are the eye-guided hysteresis loop of the FeNi layer for $30 \mathrm{~ns}$ pulses. The percentage of reversed area of the FeNi layer is given on the right axis. Circles indicate the experimental data points. (a)-(f) show the magnetic domain structure of the FeNi layer obtained by XMCD-PEEM, where the photon energy of the circularly polarized x-rays was tuned to the Fe- $\mathrm{L}_{3}$ absorption edge. Images were obtained after application of $30 \mathrm{~ns}$-short field pulses. The number and amplitude of the pulses are written below each image. The initial configurations before application of the pulses for images (a)-(c) and (d)-(f) are A and B, respectively. 

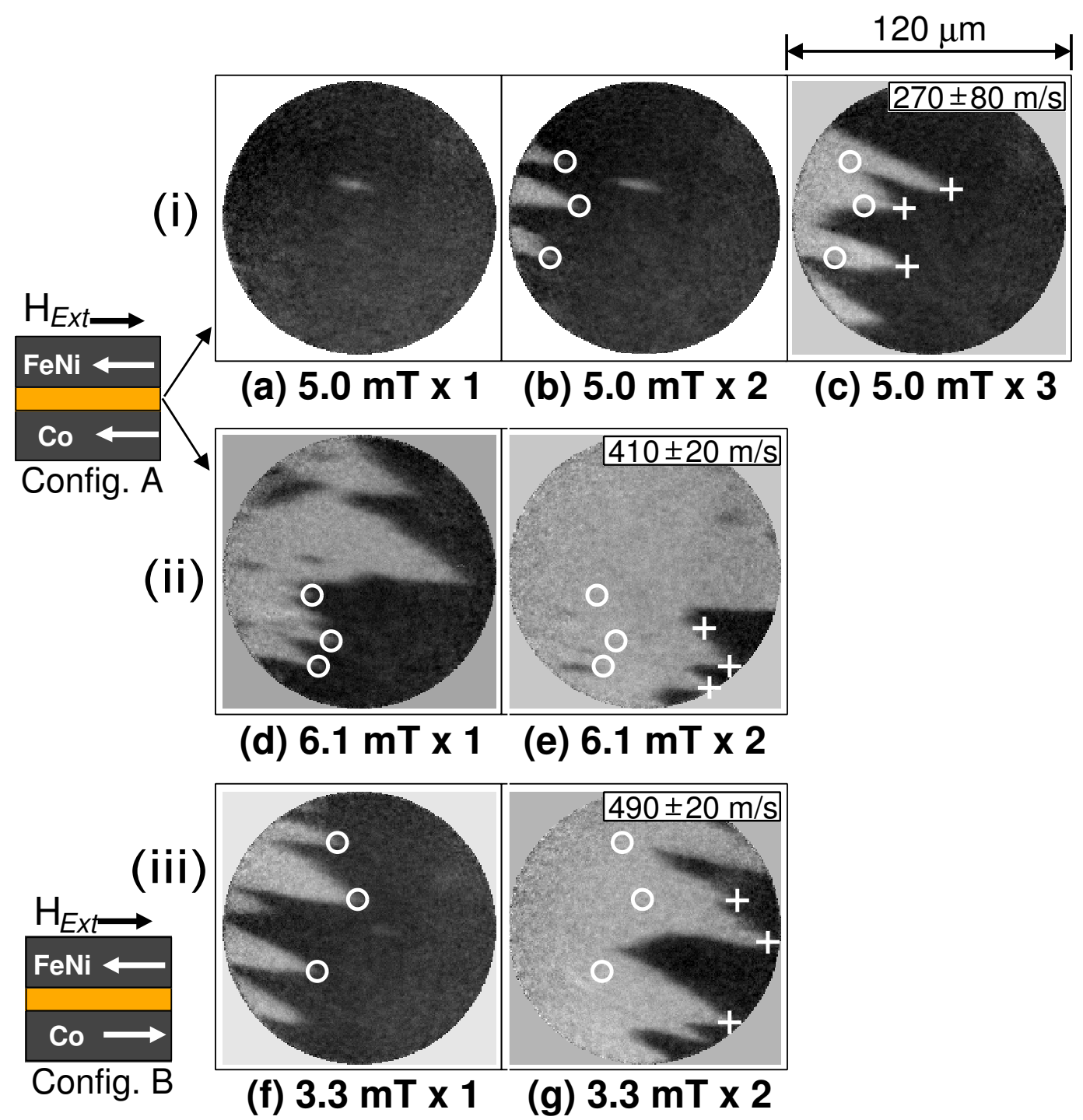

Figure 3: Magnetic domain structure of the FeNi layer. The field of view is $120 \mu \mathrm{m}$. First, the two ferromagnetic layers were either saturated in the same direction (Configuration A) or in the opposite direction (Configuration B), then 120 ns-short magnetic pulses were applied to reverse the magnetization of the FeNi layer. Images were taken after each pulse without re-saturation of the films, in order to visualize the progress of the domain walls. The number and amplitude of the applied pulses are written below each image. White circles and crosses were put onto the tips of white zig-zag domains in (b), (d) and (f) and (c), (e) and (g), respectively. The circles were superimposed from (b), (d) and (f) to (c), (e) and (g), respectively. 


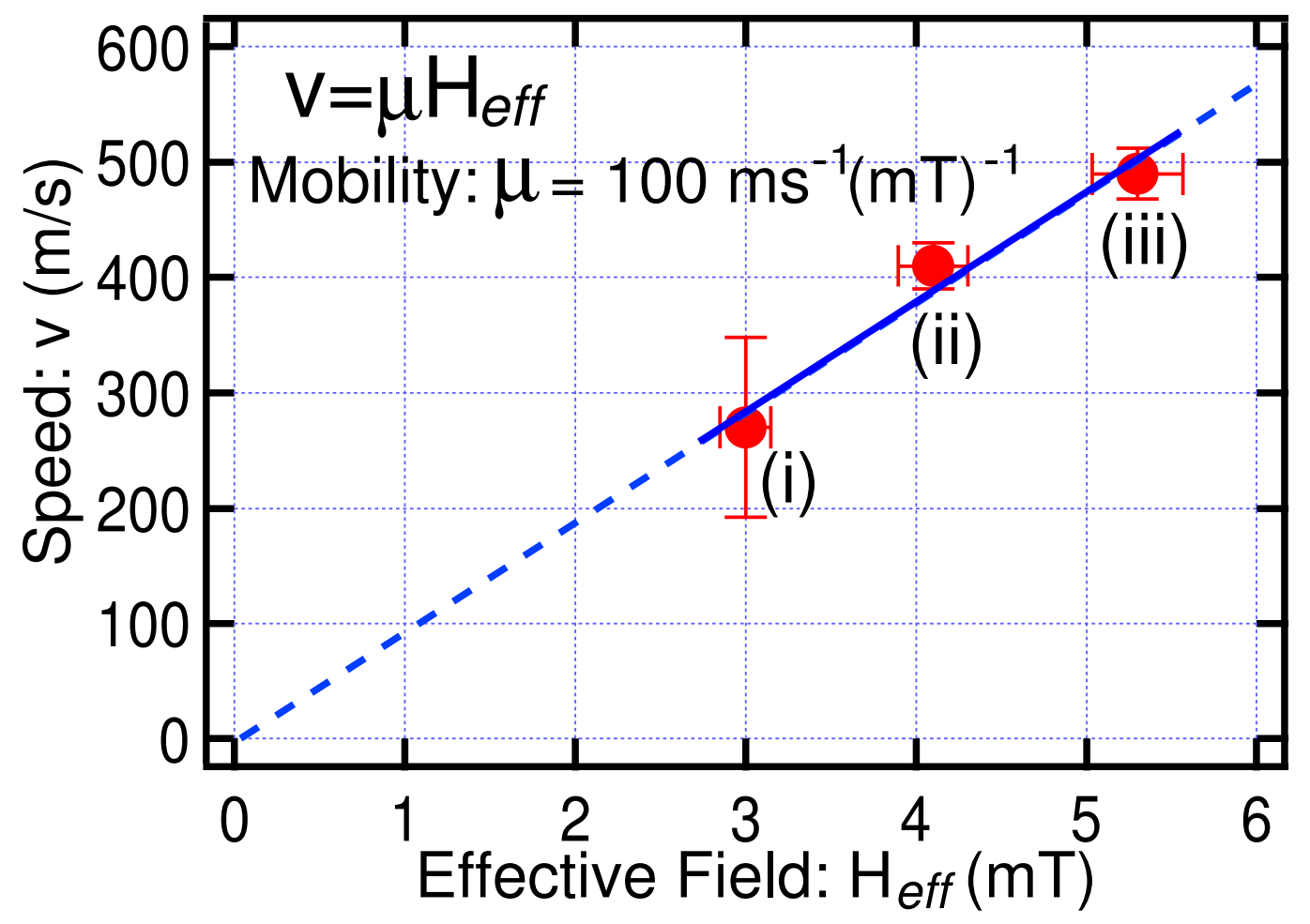

Figure 4: Domain wall speed as a function of effective field extracted from Fig. 3. The effective field was obtained by addition or subtraction of the pulse field and coupling field $(2.0 \mathrm{mT})$ for configurations B and A, respectively. Mobility $\mu$ of the wall motion was obtained by a linear fit to $\mathrm{v}=\mu \mathrm{H}_{e f f}$, which resulted in $100 \mathrm{~m} \mathrm{~s}^{-1}(\mathrm{mT})^{-1}$. 